EESTI NSV TEADUSTE AKADEEMIA TOIMETISED. XIII KÖIDE FCUSIKA-MATEMAATIKA- JA TEHNIKATEADUSTE SEERIA. 1964, NR. 4

ИЗВЕСТИЯ АКАДЕМИИ НАУК ЭСТОНСКОІІ ССР. ТОМ ХІІ СЕРИЯ ФИЗИКО-МАТЕМАТИЧЕСКИХ И ТЕХНИЧЕСКИХ НАУК. 1964, № 4

\title{
К ЭКСПЕРИМЕНТАЛЬНОМУ ОПРЕДЕЛЕНИЮ ПАРАМЕТРОВ СЛОЖНЫХ ОПТИЧЕСКИХ СИСТЕМ
}

\author{
X. АБЕН, \\ кандидат технических наук
}

В статье показывается, что метод характеристических направлений, примененный автором ранее для решения некоторых задач поляризационной оптики, применим для экспериментального определения параметров любой сптической системы, описываемой унитарной матрицей. Используя матричную символику Р. Джонса, выводятся общие соотношения метода характеристических направлений. Показывается, что характеристические величины оптической системы являются углами Эйлера соответствующего вращения сферы Пуанкаре. Дается обобщение теоремьғ эквивалентности Пуанкаре. Применение метода характеристических направлений иллюстрируется двумя примерами.

\section{Введение}

Будем рассматривать оптические системы, состоящие из поставленных друг за другом двупреломляющих пластинок и ротаторов. Ротатором называется элемент, который поворачивает плоскость поляризации света. Такие системы довольно часто встречаются в поляризационной оптике.

Обычная постановка вопроса при исследовании сложных оптических систем связана с предположением о том, что параметры отдельных элементов системы известны и требуется определить поляризацию выходящего из системы света, когда известна поляризация падающего света. Для решения этой задачи широко применяется матричный метод, разработанный Р. Джонсом $\left[{ }^{1-4}\right]$.

Однако часто необходимо решать обратную задачу - по наблюдаемым оптическим явлениям определить параметры оптической системы. Последняя задача в обшем виде в литературе не рассматривалась.

В работе автора [5] была разработана теория характеристических направлений оптических систем, которая впоследствии применялась при решении различных задач поляризационной оптики [6-9]. Целью настоящей статьи является обобщение результатов, полученных ранее, на случай любой оптической системы рассматриваемого класса. Оказывается, что, используя матричную символику Р. Джонса, можно в общем виде представить алгоритм для определения параметров оптических систем на основе экспериментально определенных характеристических величин. 


\section{Матричный метод Р. Джонса}

Состояние поляризации света определяется двумя перпендикулярными компонентами электрического вектора светового колебания. P. Джонс показал [ $\left.{ }^{1}\right]$, что при просвечивании двупреломляющей пластинки поляризованным светом компоненты светового вектора падающего света $E_{x 0}, E_{y 0}$ преобразуются в компоненты выходящего света $E_{x}, E_{y}$ соотношением

$$
\left(\begin{array}{l}
E_{x} \\
E_{y}
\end{array}\right)=\mathrm{S}(\varrho) \mathrm{G}(\gamma) \mathrm{S}(-\varrho)\left(\begin{array}{l}
E_{x 0} \\
E_{y 0}
\end{array}\right)=\mathbf{M}\left(\begin{array}{l}
E_{x 0} \\
E_{y 0}
\end{array}\right)
$$

где

$$
\mathbf{G}(\gamma)=\left(\begin{array}{ll}
e^{i \gamma} & 0 \\
0 & e^{-i \gamma}
\end{array}\right), \quad \mathbf{S}(\varrho)=\left(\begin{array}{lr}
\cos \varrho & -\sin \varrho \\
\sin \varrho & \cos \varrho
\end{array}\right),
$$

$2 \gamma$ - разность фаз двупреломляющей пластинки, $\varrho-$ - угол между главным направлением пластинки и осью $x$. Когда необходимо учитывать гакже абсолютные фазы световых колебаний, вместо матрицы $\mathbf{G}(\gamma)$ приходится использовать матрицу $e^{i x} \mathbf{G}(\gamma)$. Обычно в задачах поляризационной оптики множитель $e^{i x}$ можно опустить.

Ротатор характеризуется матришей $\boldsymbol{M}=\mathbf{S}(\beta)$, где $\beta$ - угол поворота плоскости поляризации.

Если оптическая система содержит $n$ различных элементов, то компоненты светового вектора преобразуются соотношением

$$
\left(\begin{array}{l}
E_{x} \\
E_{y}
\end{array}\right)=\mathbf{M}_{n} \mathbf{M}_{n-1} \ldots \mathbf{M}_{2} \mathbf{M}_{1}\left(\begin{array}{c}
E_{x 0} \\
E_{y 0}
\end{array}\right)=\mathbf{M}^{(n)}\left(\begin{array}{l}
E_{x 0} \\
E_{y 0}
\end{array}\right),
$$

где $\mathbf{M}_{i}$ - матрицы отдельных оптических элементов.

Так как оптические системы, состоящие из двупреломляющих пластинок и ротаторов, не изменяют интенсивность света, то матрица $\mathbf{M}^{(n)}$ является унитарной и унимодулярной. Унитарность и унимодулярность матрицы $\mathbf{M}^{(n)}$ следует также непосредственно из унитарности и унимодулярности матриц $\mathbf{G}$ и $\mathbf{S}\left[{ }^{1}\right]$.

Нахождение преобразования поляризации света сложной оптической системой, элементы которой известны, приводится к нахождению матрицы $\mathbf{M}^{(n)}$, являющейся произведением матриц отдельных оптических элементов. Для решения обратной задачи необходима дальнейшая разработка метода.

Отметим, что матричный метод Р. Джонса применим, если падающий свет полностью поляризован и оптическая система не содержит деполяризующих элементов. Если свет поляризован частично, то при описании оптических систем необходимо применять матрицы Мюллера $[4,10]$. Последний случай здесь не рассматривается.

\section{Метод характеристических направлений}

Как известно, экспериментально легко определить параметры отдельных оптических элементов системы - углы поворота рогаторов, главные направления и разности фаз двупреломляющих пластинок. Если же система состоит из нескольких элементов, то положение значительно осложняется. Для определения параметров оптической сис- 
темы в этом случае необходимо установить, какие экспериментальные величины можно определить при просвечивании системы, и каким образом они связаны с ее параметрами.

Переходим к решению поставленной задачи.

Докажем, что каждую унитарную унимодулярную матрицу $\mathbf{U}$ можно гривести к диагональному виду преобразованием

$$
\mathbf{S}\left(-\alpha_{*}\right) \dot{U} \mathbf{S}\left(\alpha_{0}\right)=\mathbf{G}(\varphi)=\left(\begin{array}{ll}
e^{i \varphi} & 0 \\
0 & e^{-i \varphi}
\end{array}\right)
$$

Наиболее общая унитарная унимодулярная матрица второго порядка может быть записана в виде

$$
\mathrm{U}=\left(\begin{array}{rl}
e^{i \xi} \cos \Theta & e^{i \xi} \sin \Theta \\
-e^{-i \zeta} \sin \Theta & e^{-i \xi} \cos \Theta
\end{array}\right)
$$

Производя умножение матриц в левой части соотношения (4) и требуя равенства нулю недиагональных элементов получаемой матрицы, имеем систему уравнений

$$
\begin{gathered}
\left(e^{i \xi} \sin \Theta \cos \alpha_{0}-e^{i \xi} \cos \Theta \sin \alpha_{0}\right) \cos \alpha_{*}+ \\
+\left(e^{-i \xi} \sin \Theta \sin \alpha_{0}+e^{-i \xi} \cos \Theta \cos \alpha_{0}\right) \sin \alpha_{*}=0 \\
\left(e^{-i \xi} \cos \Theta \sin \alpha_{0}-e^{-i \zeta} \sin \theta \cos \alpha_{0}\right) \cos \alpha_{*}- \\
-\left(e^{i \xi} \cos \Theta \cos \alpha_{0}+e^{i \zeta} \sin \Theta \sin \alpha_{0}\right) \sin \alpha_{*}=0
\end{gathered}
$$

Решением системы (6) относительно углов $\alpha_{0}$ и $\alpha_{*}$ является

$$
\begin{aligned}
\operatorname{tg} 2 \alpha_{0} & =\frac{\sin (\zeta+\xi) \sin 2 \theta}{\sin 2 \xi \cos ^{2} \Theta-\sin 2 \zeta \sin ^{2} \Theta}, \\
\operatorname{tg} 2 \alpha_{*} & =\frac{\sin (\zeta-\xi) \sin 2 \Theta}{\sin 2 \xi \cos ^{2} \Theta+\sin 2 \zeta \sin ^{2} \Theta} .
\end{aligned}
$$

Так как углы $\boldsymbol{\alpha}_{0}^{\prime}$ и $\alpha_{*}$ имеют действительные значения при любых $\Theta_{\text {, }}$ $\xi$ и $\zeta$, то наша теорема доказана.

Подставляя значения $\alpha_{0}$ и $\alpha_{*}$ из выражений (7) и (8) в диагональные элементы матрицы S $\left(-\alpha_{*}\right) \mathrm{US}\left(\alpha_{0}\right)$ и приравнивая ее матрице $\mathrm{G}(\varphi)$, получим

$$
\cos 2 \varphi=\frac{1}{2}[\cos 2 \xi+\cos 2 \zeta+\cos 2 \Theta(\cos 2 \xi-\cos 2 \zeta)] .
$$

Полученные результаты можно физически интерпретировать слепующим образом.

Отметим, что конкретный вид матрицы, характеризующей оптическую систему, зависит от выбора координатных осей, в которых описываются падающее и выходящее световые колебания. Умножение матрицы $\mathbf{U}$ справа на матрицу $\mathbf{S}\left(\alpha_{0}\right)$ означает, что падающее световое колебание описывается в координатах, составляющих угол $\alpha_{0}$ с координатами, в которых определена матрица U. Аналогично, умножение матрицы U слева на матрицу $\mathrm{S}\left(-\alpha_{*}\right)$ означает, что выходящее из системы колебание описывается в координатах, определенных углом $\alpha_{*}$.

Направления, определенные углом $\alpha_{0}$, называются первичными и направления, определенные углом $\alpha_{*}$ - вторичными характеристиче- 
скими направлениями оптической системы. Величина $2 \varphi$ называется характеристической разностью фаз.

Обозначая через $E_{10}, E_{20}$.компоненты падающего света на первичных характеристических направлениях $X_{0}, y_{0}$ и через $E_{1 *}, E_{2 *}$ - компоненты выходящего света на вторичных характеристических направлениях $X_{*}, y_{*}$, имеем в силу соотношения (4)

$$
\left(\begin{array}{l}
E_{1 *} \\
E_{2 *}
\end{array}\right)=\left(\begin{array}{ll}
e^{i \varphi} & 0 \\
0 & e^{-i \varphi}
\end{array}\right)\left(\begin{array}{l}
E_{10} \\
E_{20}
\end{array}\right)
$$

откуда следует

$$
E_{1 *}=E_{10} e^{i \varphi}, \quad E_{2 *}=E_{20} e^{-i \varphi} .
$$

Из соотношений (11) видно, что если падающий свет линейно поляризован в одном из первичных характеристических направлений, то выходящий из системы свет также поляризован линейно в соответствующем вторичном характеристическом направлении. Соответствующие друг другу первичное и вторичное характеристические направления называются сопряженными, причем угол а между ними выражается соотношением

$$
\operatorname{tg} 2 \alpha=\operatorname{tg} 2\left(\alpha_{*}-\alpha_{0}\right)=\frac{2 \sin 2 \Theta \cos \xi \cos \xi}{\sin ^{2} \xi-\sin ^{2} \xi-\cos 2 \Theta\left(\cos ^{2} \xi+\cos ^{2} \xi\right)}
$$

При просвечивании системы в обратном направлении, согласно Р. Джонсу [1], действует следующее соотношение

$$
\left(E_{x 0}^{\prime}, E_{y 0}^{\prime}\right)=\left(E_{x}^{\prime}, E_{y}^{\prime}\right) \mathbf{M}^{(n)}
$$

тде $\left(E_{x}^{\prime}, E_{y}^{\prime}\right)$ - вектор-строка падающего на $n$-й элемент системы света, $\left(E_{x 0}^{\prime}, E_{y 0}^{\prime}\right)$ - вектор-строка выходящего из первого элемента света ${ }^{1}$.

Переходя в соотношении (13) от вектор-строк к вектор-столбцам, получим

$$
\left(\begin{array}{l}
E_{x 0}^{\prime} \\
E_{y 0}^{\prime}
\end{array}\right)=\widetilde{M}^{(n)}\left(\begin{array}{l}
E_{x}^{\prime} \\
E_{y}^{\prime}
\end{array}\right),
$$

где тильда обозначает транспонирование матрицы.

Из соотношений (10) и (14) видно, что' характеристические направления сохраняют свои свойства также при просвечивании системы в обратном направлении, т. е.

$$
\left(\begin{array}{l}
E_{10}^{\prime} \\
E_{20}^{\prime}
\end{array}\right)=\left(\begin{array}{ll}
e^{i \varphi} & 0 \\
0 & e^{-i \varphi}
\end{array}\right)\left(\begin{array}{l}
E_{1 *}^{\prime} \\
E_{2 *}^{\prime}
\end{array}\right) .
$$

Так как в силу (14) при обратном просвечивании параметры $\zeta$ и $\Theta$ меняют знаки, то из соотношений $(7)$, (8) и (9) следует, что при обратном просвечивании первичные и вторичные характеристические направления меняют свои роли, а характеристическая разность фаз остается прежней.

1 Соотношение (13) имеет место, если ротаторы имеют вид обычной оптической активности. Если ротаторы обусловлены эффектом Фарадея, то соотношение (13) не действует [1]. 
Характеристические направления можно экспериментально определить при помощи любого полярископа, поляризатор и анализатор которого независимо поворачиваемы. Методика эксперимента упрощается, если использовать специальные поляризационные призмы, позволяющие установить, когда выходяший из системы свет является линейно поляризованным. Также можно использовать фотометрические методы. Характеристическая разность фаз измеряется обычными методами поляризационной оптики.

Таким образом, при просвечивании сложной оптической системь можно экспериментально определить три величины $-\alpha_{0}, \alpha_{;}$и $2 \varphi$.

На основе соотношения (4) можно матрицу оптической системы $\mathbf{U}$ выразить через характеристические величины следующим образом:

$$
\mathbf{U}=\mathbf{S}\left(\alpha_{*}\right) \mathbf{G}(\varphi) \mathbf{S}\left(-\alpha_{0}\right)
$$

Производя умножение матриц в правой части соотношения (16) и приравнивая элементы получаемой матрицы с соответствующими элементами матрицы $\mathbf{U}(5)$, получим

$$
\left.\begin{array}{l}
\operatorname{tg} \xi=\frac{\cos \left(\alpha_{0}+\alpha_{*}\right)}{\cos \left(\alpha_{0}-\alpha_{*}\right)} \operatorname{tg} \varphi, \quad \operatorname{tg} \zeta=\frac{\sin \left(\alpha_{0}+\alpha_{*}\right)}{\sin \left(\alpha_{0}-\alpha_{*}\right)} \operatorname{tg} \varphi, \\
\operatorname{tg} \Theta=\frac{\cos \xi}{\cos \zeta} \operatorname{tg}\left(\alpha_{0}-\alpha_{*}\right)=\frac{\sin \xi}{\sin \zeta} \operatorname{tg}\left(\alpha_{0}+\alpha_{*}\right) .
\end{array}\right\}
$$

Соотношения (17) позволяют по экспериментально определенным характеристическим величинам вычислять элементы матрицы оптической системы. Этот метод значительно проще метода, предложенного P. Джонсом [4], который требует просвечивания системы в обоих направлениях.

Так как элементы матрицы U зависят от длины волны света, то от нее зависят также характеристические величины оптической системы. Это явление называется дисперсией характеристических величин.

Элементы матрицы оптической системы $\xi$, $\zeta$ и $\Theta$ зависят от параметров отдельных элементов системы. Этฺ можно использовать для опреЋеления некоторых из последних (см., напр., [8]). Так как экспериментально определенных элементов матрицы три, то максимально можно определить три параметра системы. Используя дисперсию характеристических величин, т. е. производя эксперимент при различных длинах волны, можно увеличить количество определяемых параметров.

Из соотношения (1) следует, что матрицу одной двупреломляющей пластинки $\mathbf{M}^{(1)}$ можно привести к днагональному виду преобразованием

$$
S(-\varrho) M^{(1)} S(\varrho)=G(\gamma)
$$

Следовательно, сопряженные характеристические направления в: данном случае коллинеарны и совпадают с главными направлениями пластинки $\left(\alpha_{0}=\alpha_{*}=Q\right)$, а характерисгической разностью фаз является разность фаз пластинки $(\varphi=\gamma)$.

В случае, когда система состоит из одного ротатора, имеем

$$
S\left(-\alpha_{*}\right) S(\beta) S\left(\alpha_{0}\right)=G(\varphi)
$$

Так как группа врашений в плоскости является коммутативной, то можем записать

$$
\mathbf{S}\left(\alpha_{0}-\alpha_{*}\right) \mathbf{S}(\beta)=\mathbf{G}(\varphi)
$$


Из последнего соотношения получим

$$
\alpha_{*}-\alpha_{0}=\beta, \quad \mathbf{G}(\varphi)=\mathbf{E},
$$

где $\mathrm{E}$ - единичная матрица. Из изложенного следует, что характеристические направления ротатора являются неопределенными, а угол между сопряженными характеристическими направлениями равняется углу поворота ротатора. Характеристическая разность фаз ротатора равна нулю.

Из вышеизложенного следует, что метод характеристических направлений является обобщением метода, используемого при исследовании простых оптических элементов, на сложные системы, состояшие из лроизвольной комбинации последних.

\section{Обобщение теоремы эквивалентности Пуанкаре}

Согласно теореме эквивалентности Пуанкаре [11], оптическая система, состоящая из двупреломляющих пластинок, эквивалентна системе из одной двупреломляющей пластинки и одного ротатора. Эта теорема была математически доказана Р. Джонсом и X. Хурвицем [2] для систем, состоящих из двупреломляющих пластинок и ротаторов.

Теорема эквивалентности Пуанкаре непосредственно следует из возможности представить преобразование поляризации как вращение сферы Пуанкаре, так как любое вращение сферы можно рассматривать как сумму двух вращений вокруг перпендикулярных диаметров.

Различными авторами определены параметры эквивалентной оптической системы для системы из двух двупреломляющих пластинок $[12-14]$. Во всех указанных работах предполагается, что в эквивалентной системе двупреломляюцая пластинка находится перед ротатором. В работе $\left[{ }^{15}\right]$ предполагается, что и в общем случае двупреломляющая пластинка должна находиться перед ротатором. Вместе с тем из работы $\left.{ }^{2}\right]$ следует, что порядок элементов в эквивалентной оптической системе может быть произвольным.

Ввиду того, что вращения вокруг перпендикулярных осей не являются коммутативными, параметры элементов эквивалентной оптической системы зависят от того, в каком порядке они поставлены. Этот вопрос в литературе не рассматривался.

В рассматриваемый вопрос можно ввести ясность, сформулировав теорему эквивалентности в несколько более общем виде, который следует из предыдущих рассуждений.

Имея в виду, что $\mathbf{G}\left(\gamma_{1}\right) \mathbf{G}\left(\gamma_{2}\right)=\mathbf{G}\left(\gamma_{1}+\gamma_{2}\right)$ и $\mathbf{S}\left(\varrho_{1}\right) \mathbf{S}\left(\varrho_{2}\right)=$ $=\mathrm{S}\left(\varrho_{1}+\varrho_{2}\right)$, можем соотношение (16) записать в виде

$$
\mathbf{U}=\mathbf{S}\left(\alpha_{*}\right) \mathbf{G}\left(\varphi_{2}\right) \mathbf{S}\left(-\alpha_{*}\right) \mathbf{S}\left(\alpha_{*}-\alpha_{0}\right) \mathbf{S}\left(\alpha_{0}\right) \mathbf{G}\left(\varphi_{1}\right) \mathbf{S}\left(-\alpha_{0}\right)
$$

тде

$$
\varphi_{1}+\varphi_{2}=\varphi
$$

На основе последних соотношений можем сформулировать следующую теорему: любая унитарная оптическая система эквивалентна системе из двух двупреломляющих пластинок, между которыми находится ротатор; при этом угол ротатора равняется углу между главными направлениями пластинок, а разности фаз пластинок подчинены условию (23). Параметры эквивалентной оптической системы $\alpha_{0}, \alpha_{*}$ и $\varphi$ 
определяются через элементы матрицы оптической системы по соотношениям (7) -- (9).

Рассматриваем два частных случая соотношения (22):

1) $\varphi_{1}=\varphi, \quad \varphi_{2}=0$

$$
\mathbf{U}=\mathbf{S}\left(\alpha_{*}-\alpha_{0}\right) \mathbf{S}\left(\alpha_{0}\right) \mathbf{G}(\varphi) \mathbf{S}\left(-\alpha_{0}\right),
$$

2) $\varphi_{1}=0, \quad \varphi_{2}=\varphi$,

$$
\mathrm{U}=\mathrm{S}\left(\alpha_{*}\right) \mathrm{G}(\varphi) \mathrm{S}\left(-\alpha_{*}\right) \mathrm{S}\left(\alpha_{*}-\alpha_{0}\right) .
$$

Соотношения (24) и (25) выражают в математической форме теорему эквивалентности Пуанкаре в зависимости от того, находится ли двупреломляющая пластинка эквивалентной оптической системы перед или за ротатором.

Из соотношений (24) и (25) следует, что разность фаз двупреломляющей пластинки эквивалентной системы равняется характеристической разности фаз, а угол ротатора -- углу между сопряженными характеристическими направлениями. Главными направлениями двупреломляющей пластинки являются первичные или вторичные характеристические направления, в зависимости от того, находится ли пластинка перед или за ротатором.

\section{Геометрическая интерпретация на сфере Пуанкаре}

Рассмотрим изображение прохождения поляризованного света через оптическую систему на сфере Пуанкаре $\left[{ }^{10,11}\right]$ (рис. 1).

Полюсы сферы $P_{1}$ и $P_{2}$ изображают лево-круговой и право-круговой свет соотв.тственно, экватор - линейно поляризованный свет. Другие точки сферы изображают свет, который поляризован эллиптически. Диаметрально противоположные точки изображают ортогональные колебания, т. е. световые эллипсы с одинаковыми эксцентриситетами, но перпендикулярными длинными осями и противоположными направлениями вращения светового вектора. Радиус сферы равен единице.

Произвольно выбранные точки $X, У$ на экваторе, находящиеся на одном диаметpe, изображают координат-

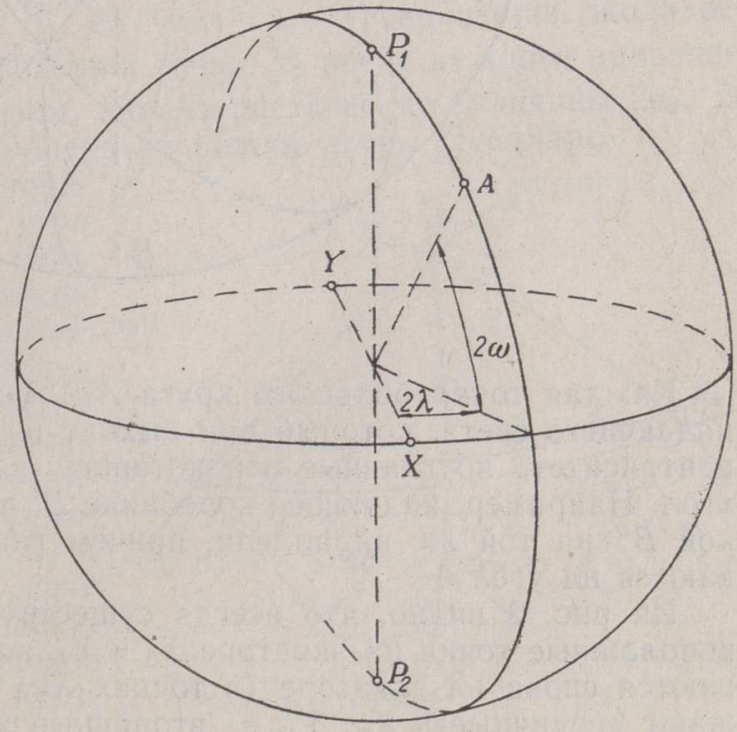

Рис. 1. ные оси, относительно которых описывается свеговой эллипс. Точка $A$ с долготой $2 \lambda$ и широтой $2 \omega$ изображает световой эллипс с азимутом $\lambda$ относительно оси $X$ и с эксцентриситетом $\omega$.

Прохождение света через сложную оптическую систему изображается вращением сферы вокруг ее центра. При этом, если свет проходит 
двупреломляюшую пластинку, то ось вращения находится в экваториальной плоскости и угол врашения равняется разности фаз пластинки; если же свет проходит ротатор, то вращение производится вокруг оси $P_{1} P_{2}$ на удвоенный угол ротатора.

Будем рассматривать вращение сферы, которое соответствует влиянию оптической системы как целой. Согласно теореме Эйлера $\left.{ }^{16}\right]$, любое вращение тела, имеющего неподвижную точку, можно осуществлять посредством вращения вокруг некоторой неподвижной оси. Обозначаем следы оси вращения на сфере $A_{1}, A_{2}$ и угол поворота сферы $\delta$ (рис. 2). Жзображаем большой круг $A_{1} A^{\prime} A_{2}$, который при поворачивании около оси $A_{1} A_{2}$ на угол $\delta$ приобретает симметричное относительно меридиана $A_{1} P_{1} A_{2} P_{2}$ положение $A_{1} A^{\prime \prime} A_{2}$.

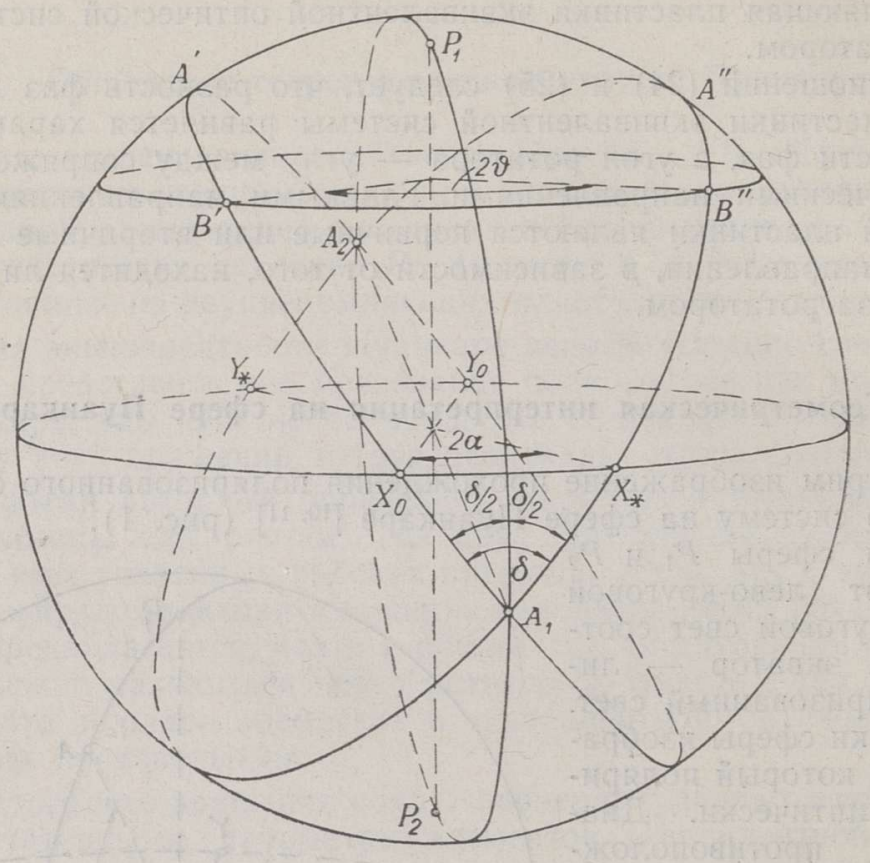

Рнс. 2.

Каждая точка большого круга $A_{1} A^{\prime} A_{2}$ изображает световой эллипс падающего света, который при выходе из системы имеет прежний эксцентриситет, но главные оси которого поворачиваются на некоторый угол. Например, падающее колебание $B^{\prime}$ при выходе изображается точкой $B^{\prime \prime}$ на той же параллели, причем главные оси эллипса поворачиваются на угол $\vartheta$.

Из рис. 2 видно, что всегда существуют две диаметрально противоположные точки на экваторе $X_{0}$ и $Y_{0}$, которые после поворота оказываются снова на экваторе (в точках $X_{*}, Y_{*}$ ). Точки $X_{0}$ и $Y_{0}$ изображают первичные и $X_{*}, Y_{*}$ - вторичные характеристические направления оптической системы. Так как колебание $X_{0}$ преобразуется в колебание $X_{*}$, то $X_{0}$ и $X_{*}$ являются сопряженными характеристическимн направлениями; сопряженными являются также $Y_{0}$ и $Y_{*}$. Таким образом, существование характеристических направлений непосредственно следует из теоремы Эйлера.

Покажем теперь, что характеристические величины оптической системы являются углами Эйлера врашения сферы Пуанкаре. 
Изображаем вращение сферы начальным и конечным положением экватора. Из рис. 3 видно, что экватор $X A^{\prime} Y$ можно перевести в конечное положение $X_{:} A^{\prime \prime} y_{:}$следующими вращениями: 1) вращение около оси $P_{1} P_{2}$ на угол $2 \alpha_{: ;}$; это вращение совмещает ось $X Y$ с осью $X_{*} Y_{: \text {: }}$

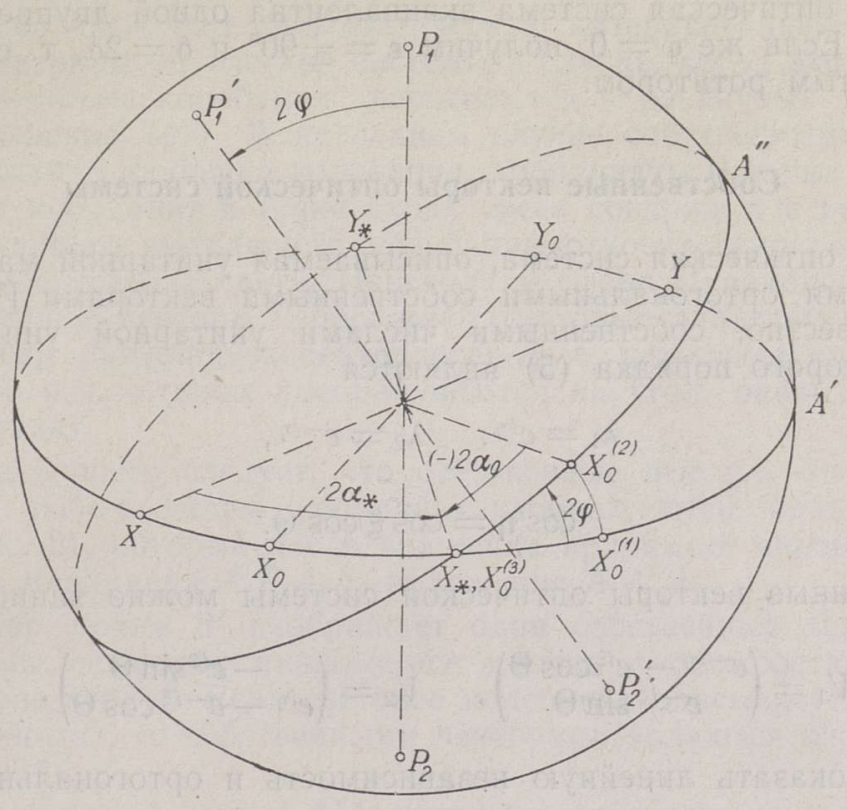

Рис. 3.

2) вращение около оси $X_{*}, Y_{:}$на угол $\left.{ }^{2} 2 \varphi ; 3\right)$ вращение около оси $P_{1}^{\prime} P_{2}^{\prime}$ на угол $\left(-2 \alpha_{0}\right)$. Положения точки $X_{0}$ после каждого вращения обозначены $X_{0}^{(i)}$. Таким образом, характеристические величины $2 \alpha_{\%}, 2 q$ и $\left(-2 \alpha_{0}\right)$ являются углами Эйлера вращения сферы Пуанкаре.

Определяем через характеристические величины ось и угол вращения сферы Пуанкаре. Обозначаем сопряженные характеристические направления через $X_{0}$ и $X_{*}$ (рис. 4). Так как точка $X_{0}$ после вращения переводится в точку $X_{*}$, то след оси вращения $A_{1}$ должен находиться на меридиане $B K$, расположенном симметрично относительно точек $X_{0}$ и $X_{*}$ Изображхам большой круг $X_{0} K A^{\prime}$, который врашением около $A_{1}$ приводится в симметричіое относительно меридиана $K B A_{1}$ положение $X_{\%} K A^{\prime \prime}$.

Обозначаем широту оси вращения $\varepsilon$ и угол поворота сферы $\delta$.

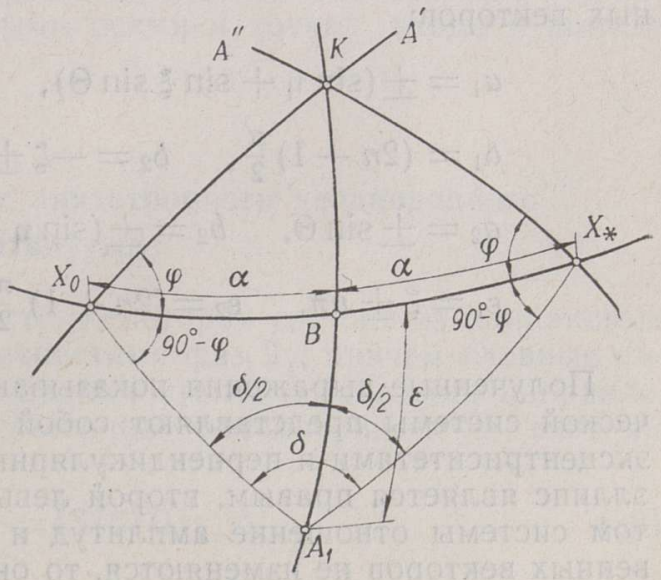

Рис. 4. Нз прямоугольного сферического треугольника $A_{1} B X_{0}$ или $A_{1} B X_{*}$ легко получить соотношения

2. Из теоремы эквивалентности следует, чго конечное положение экваториальной плоскости составляет с начальным положением угол $2 \varphi$. 


$$
\operatorname{tg} \varepsilon=\sin \alpha \operatorname{ctg} \varphi, \quad \cos \frac{\delta}{2}=\cos \alpha \cos \varphi
$$

Легко проверить справедливость формул (26) в некоторых простых частных случаях. Если $\alpha=0$, то $\varepsilon=0$ и $\delta=2 \varphi$. Это соответствует случаю, когда оптическая система эквивалентна одной двупреломляющей пластинке. Если же $\varphi=0$, получим $\varepsilon= \pm 90^{\circ}$ и $\delta=2 \alpha$, т. е. мы имеем дело с чистым ротатором.

\section{Собственные векторы оптической системы}

Каждая оптинеская система, описываемая унитарной матрицей, обладает двумя ортогональными собственными векторами [3, 10].

Как известно, собственными тислами унитарной унимодулярной матрицы второго порядка (5) являются

$$
\lambda_{1}=e^{i \eta}, \quad \lambda_{2}=e^{-i \eta},
$$

где

$$
\cos \eta=\cos \xi \cos \Theta
$$

Собственные векторы оптической системы можно записать в виде

$$
V_{1}=\left(\begin{array}{c}
e^{-i \eta}-e^{i \xi} \cos \theta \\
e^{-i \zeta} \sin \theta
\end{array}\right), \quad V_{2}=\left(\begin{array}{c}
-e^{i \zeta} \sin \theta \\
e^{i \eta}-e^{-i \xi} \cos \theta
\end{array}\right)
$$

Легко доказать линейную независимость и ортогональность векторов $V_{1}$ и $V_{2}$.

Выражая собственные векторы в виде

$$
V_{1}=\left(\begin{array}{ll}
a_{1} & e^{i \hat{o}_{1}} \\
b_{1} & e^{i \hat{o}_{2}}
\end{array}\right), \quad V_{2}=\left(\begin{array}{ll}
a_{2} & e^{i \varepsilon_{1}} \\
b_{2} & e^{i \varepsilon_{2}}
\end{array}\right)
$$

можем, используя соотношения (29), определить параметры собственных векторов:

$$
\begin{aligned}
& c_{\mathrm{i}}= \pm(\sin \eta+\sin \xi \sin \theta), \quad b_{1}= \pm \sin \theta, \\
& \delta_{1}=(2 n+1) \frac{\pi}{2}, \quad \delta_{2}=-\xi \pm n \pi, \quad(n=0,1,2, \ldots) \\
& a_{2}= \pm \sin \Theta, \quad b_{2}= \pm(\sin \eta+\sin \xi \sin \theta), \\
& \varepsilon_{1}=\xi \pm n \pi, \quad \varepsilon_{2}=(2 n+1) \frac{\pi}{2}
\end{aligned}
$$

Полученные выражения показывают, что собственные векторы оптической системы представляют собой два световых эллипса с равными эксцентриситетами и перпендикулярными главными осями, причем один эллипс является правым, второй левым. Так как при прохождении светом системы отношение амплитуд и разность фаз компонентов собственных векторов не изменяются, то они представляют поляризацию света, которая не изменяется при прохождении системы. Так, собственными векторами двупреломляющей пластинки являются колебания, линейно поляризованные в главных направлениях пластинки; собственными векторами ротатора являются колебания, поляризованные по кругу. В общем случае собственными векторами оптической системы являются следы оси вращения $A_{1}$ и $A_{2}$ (рис. 2). 
Называя стношение амплитуд и разность фаз компонентов светового вектора его координатами, можні) сказать, что собственными векторами являются световые эллипсы, координаты которых не изменяются при прохождении системы. Это относится, конечно, к состояниям поляризации при входе и выходе.

Сказанное позволяет дать физнческую интерпретацию собственным векторам матрицы оптической системы в случае, когда входящие и выходящие световые колебания описываются в различных координатах (см. соотношение (4)). В последнем случае собственными векторами являются такие падающие колебания, координаты которых относительно системы координат в точке входа света совпадают с координатами соответствующего выходящего колебания относительно системы координат в точке выхода света. Переходя к сфере Пуанкаре, это значит, что собственные векторы изображают световые эллипсы, которые при выходе имеют прежний эксцентриситет, т. е. находятся на той же параллели, но оси которых поворачиваются на угол, равный углу поворота координат.

Из предыдущего следует, что собственные векторы оптической системы для любых систем координат изображаются большим кругом $A_{1} A^{\prime} A_{2}$ (рис. 2), который после вращения принимает симметричное относительно меридиана $A_{1} P_{1} A_{2} P_{2}$ положение $A_{1} A^{\prime \prime} A_{2}$.

Например, точка $B^{\prime}$ изображает один собственный вектор оптической системы, если координатные оси в точке выхода составляют с осями при входе угол $\vartheta$. Если световое колебание описывается в постоянных координатах, то собственными векторами являются следы оси вращения $A_{1}$ и $A_{2}$.

Так как большой круг $A_{1} A^{\prime} A_{2}$ непременно пересекает экватор в двух диаметрально противоположных точках $X_{0}$. и $Y_{0}$, то при некотором угле поворота координат $\alpha$ собственные векторы являются линейно поляризованными.

Следовательно, первичные характеристические направления можно интерпретировать как линейно поляризованные собственные векторы, которыми обладает любая унитарная оптическая система при определенном угле между координатными осями в точках входа и выхода света.

\section{Частный случай оптически анизотропного неоднородного континуума}

Рассматриваем оптическую систему, которая состоит из одинаковых двупреломляющих пластинок с разностями фаз $2 \gamma$, причем главные направления каждой пластинки составляют угол $\beta$ с главными направлениями предыдушей пластинки. Матрица рассматриваемой системы имеет вид

$$
\mathbf{M}^{(n)}=[\mathbf{S}(\beta) \mathbf{G}(\gamma)]^{n}
$$

Отметим, что координатными осями в точке входа света являются главные направления первой пластинки, а координатные оси в точке выхода света составляют угол $\beta$ с главными направлениями последней плластинки.

Матрица $\mathbf{M}^{(n)}$ имеет в данном случае вид 


$$
\mathbf{M}^{(n)}=\left(\begin{array}{ll}
\cos n \chi+ & \frac{\sin n \chi}{\sin \chi} \cos \gamma \sin \beta- \\
\frac{1}{-} i \frac{\sin n \chi}{\sin \chi} \sin \gamma \cos \beta & -i \frac{\sin n \chi}{\sin \chi} \sin \gamma \sin \beta \\
-\frac{\sin n \chi}{\sin \chi} \cos \gamma \sin \beta- & \cos n \chi-i \frac{\sin n \chi}{\sin \chi} \sin \gamma \cos \beta \\
-i \frac{\sin n \chi}{\sin \chi} \sin \gamma \sin \beta & -i
\end{array}\right)
$$

где $\cos \chi=\cos \gamma \cos \beta$.

Пусть чнсло двупреломляющих пластинок стремится к бесконечности при фиксированных значениях суммарной разности фаз $2 \gamma_{n}$ и суммарного угла поворота главных направлений $\beta_{n}\left(\gamma=\frac{\gamma_{n}}{n}, \quad \beta=\frac{\beta_{n}}{n}\right)$. Тогда матрица $\mathbf{M}^{(n)}$ будет описывать оптически анизотропную неоднородную сплошную среду с равномерным врацением главных направлений при постоянном значении двупреломления.

На основе выражения (33) находим

$$
\lim _{n \rightarrow \infty} \mathbf{M}^{(n)}=\left(\begin{array}{cc}
\cos \psi+i \frac{1}{S} \sin \psi & \frac{R}{S} \sin \psi \\
-\frac{R}{S} \sin \psi & \cos \psi-i \frac{1}{S} \sin \psi
\end{array}\right)
$$

Здесь приняты обозначения

$$
\frac{\beta_{n}}{\gamma_{n}}=R, \quad \sqrt{1+R^{2}}=S, \quad S \gamma_{n}=\psi .
$$

Приравнивая соответствующие элементы матриц (5) и (34), получим систему уравнений

$$
\left.\begin{array}{ll}
\cos \xi \cos \Theta=\cos \psi, & \sin \xi \cos \theta=\frac{1}{S} \sin \psi, \\
\cos \xi \sin \Theta=\frac{R}{S} \sin \psi, & \sin \xi \sin \Theta=0 .
\end{array}\right\} .
$$

\section{Решением системы. (36) является}

$$
\operatorname{tg} \xi=\frac{1}{S} \operatorname{tg} \psi, \quad \xi=m \pi, \quad \sin \Theta=(-1)^{m} \frac{R}{S} \sin \psi, \quad(m=0,1,2 \ldots)
$$

Подставляя полученные результаты в формулы (7) - (9) и (12), находим выражения для определения характеристических величин рассматриваемой оптической системы

$$
\begin{gathered}
\operatorname{tg} 2 \alpha_{0}=\frac{R}{S} \operatorname{tg} \psi, \quad \operatorname{tg} 2 \alpha_{*}=-\frac{R}{S} \operatorname{tg} \psi \\
\cos 2 \varphi=1-\frac{2}{S^{2}} \sin ^{2} \psi \\
\operatorname{tg} \alpha=\operatorname{tg}\left(\beta_{n}+\alpha_{*}-\alpha_{0}\right)=\frac{\operatorname{tg} \beta_{n}-\frac{R}{S} \operatorname{tg} \psi}{1+\frac{R}{S} \operatorname{tg} \beta_{n} \operatorname{tg} \psi} .
\end{gathered}
$$


Рассматриваемая задача представляет определенный интерес в методе фотоупругости. В объемных фотоупругих моделях часто имеет место вращение главных напряжений. Так как направления главных напряжений совпадают с главными направлениями оптической анизотропии, то фотоупругие модели представляют собой оптические системы с вращающимися главными направлениями. Иногда это вращение можно считать равномерным при постоянной величине оптической анизотропии.

Исходя из решения дифференциальных уравнений фотоупругости, формулы (38) были получены автором в работе [17] другим путем. Приведенный пример показывает, что интегрированне уравнений фотоупругости эквивалентно нахождению предела матрицы соответствуюшей дискретной оптической системы. Следовательно, теория характери стических направлений в матричном представлении может оказаться полезной также при исследовании анизотропного неоднородного континуума.

\section{Среда с наложенными свойствами двупреломления и оптической активности}

Рассматриваем оптическую систему, которая состоит из одинаковых двупреломляющих пластинок, имеющих параллельные главные направления и разности фаз $2 \gamma$. Между пластинками находятся одинаковые ротаторы с углами поворота $\beta$. Такая оптическая система описывается также матрицей (32), а в случае, когда число элементов системы стремится к бесконечности, матрицей (34). В последнем случае мы имеем дело со средой с наложенными свойствами двупреломления и оптической активности. Необходимо лишь иметь в виду, что теперь световые колебания в точке входа и выхода описываются в постоянных координатах, совпадающих с главными направлениями двупреломления.

Из соотношений (38) следует, что сопряженные характеристические направления расположены симметрично относительно главных направлений двупреломления, т. е. последние являются биссектрисой угла $\alpha$, 'Который выражается в виде

$$
\operatorname{tg} \alpha=\operatorname{tg}\left(\alpha_{*}-\alpha_{0}\right)=-\frac{R}{S} \operatorname{tg} \psi
$$

Определяя экспериментально характеристические величины, можно из соотношений (38) и (39) определить двупреломление $2 \gamma_{n}$ и угол поворота $\beta_{n}$ среды. Этот метод несколько проще методов, предложенных лругими авторами $\left[{ }^{18-20}\right]$.

Матрица (34) вместе с соотношениями (38) и (39) полностью описывают оптические свойства оптически активной двупреломляющей среды. Например, формула (39) показывает, как изменяется наблюдаемый «угол поворота» плоскости поляризации в зависимости от двупреломления и оптической активности, а третье соотношение (38) показывает зависимость измеряемой характеристической разности фаз от тех же величин.

Л И Т Е Р А Г У А

1. J ones R. C., J. Opt. Soc. Amer., 31, No. 7, 488-493, 500-503 (1941).

2. Hurwitz H., Jones R. C., J. Opt. Soc. Amer., 31, No. 7, 493-499 (1941).

3. Jon es R. C., J. Opt. Soc. Amer., 32, No. 8, 486-493 (1942). 
4. Jones R. C., J. Opt. Soc. Amer., 37, No. 2, 107-112 (1947).

5. А б е н Х., Изв. АН Эст. ССР. Сер. физ-матем. и техн. наук, 11, № 1, 16-23 (1962).

6. А б е н X. К., Опт. и спектр., 13, № 5, 746-750 (1962).

7. А б е н Х. Қ., Опт. и спектр., 14, № 2, 240-246 (1963).

8. А б е н Х. К., Опт. и спектр., 15, № 5, 682-689 (1963).

9. А б е н Х., Изв. АН Эст. ССР. Сер. физ-матем. и техн. наук, 12, № 4, 369-375 (1963).

10. Schurcliff W. A., Polarized light, Cambridge, Massachusetts, 1962.

11. P o in c a ré H., Théorie mathématique de la lumière, II, Paris, 1892.

12. R i ch a r tz M., H s i e n-Yü Hsü, J. Opt. Soc. Amer., 39, No. 2, 136-157 (1949).

13. Le r a y, J., S che ibling G., S a d r o n Ch., Compt. Rend., 252, No. 12, 1748-1749 (1961).

14. Ler a y, J., S che ibling G., J. Chim. Phys., 58, No. 9, 797-802 (1961).

15. Robert A., Mme Guill e me t, Rev. Franç. de Mécanique, Nr. 5/6, 147-157 (1963).

16. Голдст ейн Г., Классическая механика, М., 1957.

17: А б ен Х. К., Изв. АН СССР, ОТН, Механика и машиностроение, № 3, 141-147 (1962).

18. Ma 11 e m a n n R., Compt. Rend., 176, 380-382 (1923).

19. R a m a chandran G. N., Chandrasekharan V., Proc. Indian Acad. Sci., 33, A, No. 3, 199-215 (1951).

20. R a m a ch a ndran G. N., R a m a se sh a n S., J. Opt. Soc. Amer., 42, No. 1, 49-56 (1952).

Институт кибернетики

Академии наук Эстонской ССР

Поступила в редакцию

11 III 1964

\title{
KEERUKATE OPTILISTE SÜSTEEMIDE PARAMEETRITE EKSPERIMENTAALSEST MÄARRAMISEST
}

\author{
H. Aben, \\ tehnikateaduste kandidaat
}

Resümee

Artiklis näidatakse, et karakteristlike suundade meetod, mida autor on varem kasuta. nud mõningate polarisatsioonoptika ülesannete lahendamiseks $\left[{ }^{5-9}\right]$, on rakendatav kõigi unitaarsete maatriksitega kirjeldatavate optiliste süsteemide parameetrite määramiseks. Optilise süsteemi karakteristlikud suurused $\alpha_{0}, \alpha_{*}$ ja $\varphi$ avalduvad süsteemi maatriksi parameetrite kaudu seostega (7)-(9). Süsteemi maatriksi parameetrid leitakse eksperimentaalselt määratud karakteristlike suuruste abil seostest (17).

Optiliste süsteemide ekvivalentsusteoreem formuleeritakse üldises kujus (22), millest erijuhtudena tulenevad Poincaré ekvivalentsusteoreemi mõlemad võimalikud kujud (24) ja (25).

Esitatakse karakteristlike suundade meetodi interpretatsioon Poincaré' sfääril, kusjuures näidatakse, et optilise süsteemi karakteristlikud suurused on vastava Poincaré sfääri pöörde Euleri nurkadeks.

Tuletatakse seosed (31) optilise süsteemi omavektorite komponentide määramiseks ning näidatakse, et iga unitaarne optiline süsteem omab teatud koordinaadistiku pöörde puhul lineaarselt polariseeritud omavektoreid, mille vônkesuunad ongi süsteemi primaarseteks karakteristlikeks suundadeks.

Tuletatakse maatriks (34), mis kirjeldab optiliselt anisotroopset mittehomogeenset. keskkonda anisotroopia peatelgede ühtlase pöördumise puhul, ning seosed (38) vaadeldava süsteemi karakteristlike suuruste määramiseks. Näidatakse, et erinevas koordinaadistikus, kirjeldab maatriks (34) ka optiliselt aktiivse kaksikmurdva keskkonna optilisi omadusi.
Eesti NSV Teaduste Akadeemia Küberneetika Instituut
Saabus toimetusse
11. III 1964 


\section{ON THE EXPERIMENTAL DETERMINATION OF PARAMETERS OF COMPLEX OPTICAL SYSTEMS}

\section{H. Aben}

\section{Summary}

It is shown that the method of characteristic directions, which was previously applied by the author $\left[^{5-9}\right]$ in solving some problems of polarization optics, can be used in determining of parameters of all optical systems which can be described by unitary matrices. The characteristic parameters of the optical system $\alpha_{0}, \alpha_{*}$ and $\varphi$ are determined through the elements of the matrix of the optical system by formulae (7)-(9). The elements of the matrix can be found from formulae (17) if the characteristic parameters are determined experimentally.

The equivalence theorem of optical systems is formulated in a general form (22), from which the two possible formulations of Poincaré's equivalence theorem (24) and (25) follow.

An interpretation of the method of characteristic directions on Poincaré's sphere is presented and it is shown that the characteristic parameters of an optical system are Eulerian angles of the rotation of Poincaré's sphere.

Formulae (31) allowing to determine the components of eigenvectors of optical systems are derived. It is shown that by a certain rotation of coordinate axes every unitary optical system possesses linearly polarized eigenvectors which determine the primary characteristic directions of the system.

A matrix (34) is derived, which describes a birefringent inhomogeneous medium when the principal axes of birefringence rotate uniformly. Characteristic paramaters of the system are determined by the formulae (38). Using another coordinate system, the same matrix (34) describes optical properties of birefringent and optically active media.
Academy of Sciences of the Estonian S.S.R.,
Received Institute of Cybernetics 